\title{
Contrast Enhancement of Colour Images by Optimized Fuzzy Intensification
}

\author{
Avadhesh Kumar Dixit ${ }^{1}$, Rakesh Kumar Yadav ${ }^{2}$ and Ramapati Mishra ${ }^{3}$ \\ ${ }^{1}$ Research Scholar, IFTM University, Moradabad, Uttar Pradesh, India \\ ${ }^{2}$ Assistant Professor, IFTM University, Moradabad, Uttar Pradesh, India \\ ${ }^{3}$ Professor, Dr. RamManohar Lohia Avadh University, Ayodhya, Uttar Pradesh, India \\ *Correspondence: Avadhesh Kumar Dixit; Email: dixit1008@gmail.com
}

\begin{abstract}
Contrast enhancement is a critical and difficult issue because inappropriate enhancement by existing global image enhancement techniques might result in over or under enhancement. Varying areas of the image that are lighted indicate different shades and contrast in the output images. Projected technique uses local colour correction in the Hue Saturation Luminance (HSL) colour space. To control colour fidelity in initial phase an optimized fuzzy intensification parameters are extracted automatically form fuzzy inference system for that particular image. Finally optimized Fuzzy Intensification parameter constants are used to minimize overexposed and underexposed areas and offers elevated contrast improvement. Several lab test conducted to analyze the effectiveness of the proposed method with existing strategies. Many quality evaluation parameters are evaluated, and findings are compared to some known colour picture contrast enhancement approaches. The produced output comparatively better than many existing techniques which support a moderate measure to visual perception of the processed images.
\end{abstract}

Keywords: Local Colour Correction, Fast Fourier Transform, Local colour correction, fuzzy intensification, Fuzzy inference system

\begin{tabular}{|c|c|}
\hline \multicolumn{2}{|l|}{ ARTICLE INFORMATION } \\
\hline \multicolumn{2}{|c|}{$\begin{array}{l}\text { Author(s): Avadhesh Kumar Dixit, Rakesh Kumar Yadav and Ramapati } \\
\text { Mishra } \\
\text { Received: Nov 11, 2021; Accepted: Feb 20, 2022; Published: Feb 28, 2022; }\end{array}$} \\
\hline $\begin{array}{l}\text { e-ISSN: } 2347-470 X \\
\text { Paper Id: IJEER120112; }\end{array}$ & Crossef member \\
\hline $\begin{array}{l}\text { Citation: doi.org/10.37391/IJEER.090408 } \\
\text { Webpage-link: }\end{array}$ & $\begin{array}{l}\text { CROSSREFOORG } \\
\text { THE CIATON LINKING BACKBONE }\end{array}$ \\
\hline
\end{tabular}

\section{INTRODUCTION}

Image enhancement is very popular area of image processing which aim is to convert the image in more acceptable viewing and have least distortion or noise. Many real life application areas like satellite imaging, geographical information system, criminal identification and tracing, medical diagnostics, astronomy and visual cryptography uses an enhanced image to achieve their target. Among many image enhancement techniques most of them are satisfying the need of particular area of application like removing blur, removing extra noise, increasing contrast, improving optimal lightness or luminance. Contrast is difference between highest and lowest intensity values of pixel. It differentiates image object with its background. Contrast depend upon many factors like camera quality, available illuminance, differentiating power of human eye and surrounding environment etc. Often resultant image is not providing sufficient details which exhibit unnatural look due to washing out some part of image.

Contrast improvement algorithm tries to remove all obstacle mentioned above and also provide optimum contrast [1][2]. Two fundamental and direct contrast measure [3][4] algorithm are Michelson Contrast Measures and Weber Contrast Measures which can be used for periodic pattern or uniform luminance background. HE technique suffers from over or under enhancement and also produces unwanted artifact in bright regions. Adaptive Histogram Equalization (AHE) [5], Dynamic Histogram Equalization (DHA) [6] improves the performance to some extent on pixel level by using local histogram but fails on non-uniform illumination images. Many others Global Histogram Equalization (GHE) [7] algorithm such as, Brightness Preserving Bi-Histogram Equalization (BPBHE) [8], BPDHE [9], DSIHE [10] and MMBEBHW [11] [12] are sensitive to noise are not able to enhance up to a level.

To Increase the contrast of low lighting colour images Fuzzy Logic and Histogram (FLS) [12] based is better but the major drawback is that it fails to enhance dark images. An Averaging Histogram Equalization Technique (AVHEQ) [13] is provided for enhancement of contrast which controls the distortion and frequency offered in system but sometimes suffers from over enhancement.

Optimal Profile Compression and HE for colour images [14] is offered which maximize saturation operation. A specific method proposed named contrast limited adaptive histogram equalization with dual gamma correction [15] is used to boost the luminance and increase contrast of image. With the help of homomorphic decomposition an improved contrast is shown by Syed Zaheeruddin et al. [16] in uniform radiance images. B. Subramani and M. Veluchamy [17] presented quadrant dynamic clipped histogram equalization method using Gamma modification to reduce excess enhancement and contains more components. The proposed method has a high level of entropy, colourfulness, and saturation, as well as a smooth enhancement. R. Chandrasekharan et al. [18], has proposed a complex parametric variation for the differential enhancement of distinctly illuminated images with enhanced nature and 
preservation details.

Analysis results show that the algorithm takes lower calculation costs. Although Retinex is a colour photography technique, it still has issues with Gray-level refinement, distorted appearances, and strange colour reproduction. After reviewing the literature, we find that there are currently some possibilities for advancing in existing comparisons with the use of local colour correction and automatic enhancement of the image operator's capabilities through an abstract thinking system.

\section{MATERIALS AND METHODS}

The suggested model is simple and effective, and it addresses fundamental flaws in existing models. This is also my extended research of [19]. The proposed method's fundamental idea is to use the image after local colour correction in the Hue Saturation Luminance (HSL) domain and then apply the default automatic fuzzy intensification. Intensification operators are employed to manage the colour fidelity of the local colour correction images, allowing them to handle the problem of over-exposed and non-highlighted regions while also improving colour image contrast adjustment. The constant fuzzy intensification adjustment may not fit a particular image so the model has found the best reinforcement operator using an incomprehensible simulation system. A great deal of research has gone into determining the efficacy of the proposed strategy as well as the feasibility of comparing previous strategies.
Fuzzy Intensification operator is used to increase image contrast and reduce fuzziness. We need two parameters to measure operational strength operators. The first $\operatorname{tau}(\tau)$ parameter defining the boundary operator. The second parameter is a membership function, which is required since the specified channel pixels modify the default distance between 0 and 1 . Membership performance measures for any channel can now be obtained by equation (1).

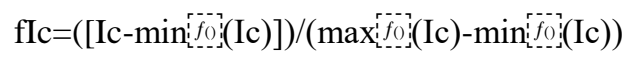

Where here c represents any colour red, green and blue and fIc represent membership function value for any channel red, green or blue. This way we calculate membership values. Now before processing the red, green and blue channel, image is passed to another function for calculating automatic fuzzy intensification operator by using fuzzy inference system.

Now role of $\tau \mathrm{c}\{\tau \mathrm{R}, \tau \mathrm{G}, \tau \mathrm{B}\}$ is very crucial for calculating processed channel by intensification operator. We generally take the $\{\tau \mathrm{R}, \tau \mathrm{G}, \tau \mathrm{B}\}$ value as scalar but scalar values may not be appropriate for every types of images. Here we are proposing spontaneous cunning of $\{\tau \mathrm{R}, \tau \mathrm{G}, \tau \mathrm{B}\}$ values with the help of fuzzy inference system. Appropriate value of $\{\tau R$, $\tau \mathrm{G}, \tau \mathrm{B}\}$ will lead to better enriched image and restore the novel content of image. Figure 1 represent the proposed model for extracting automatic fuzzy intensification operator using fuzzy inference system.

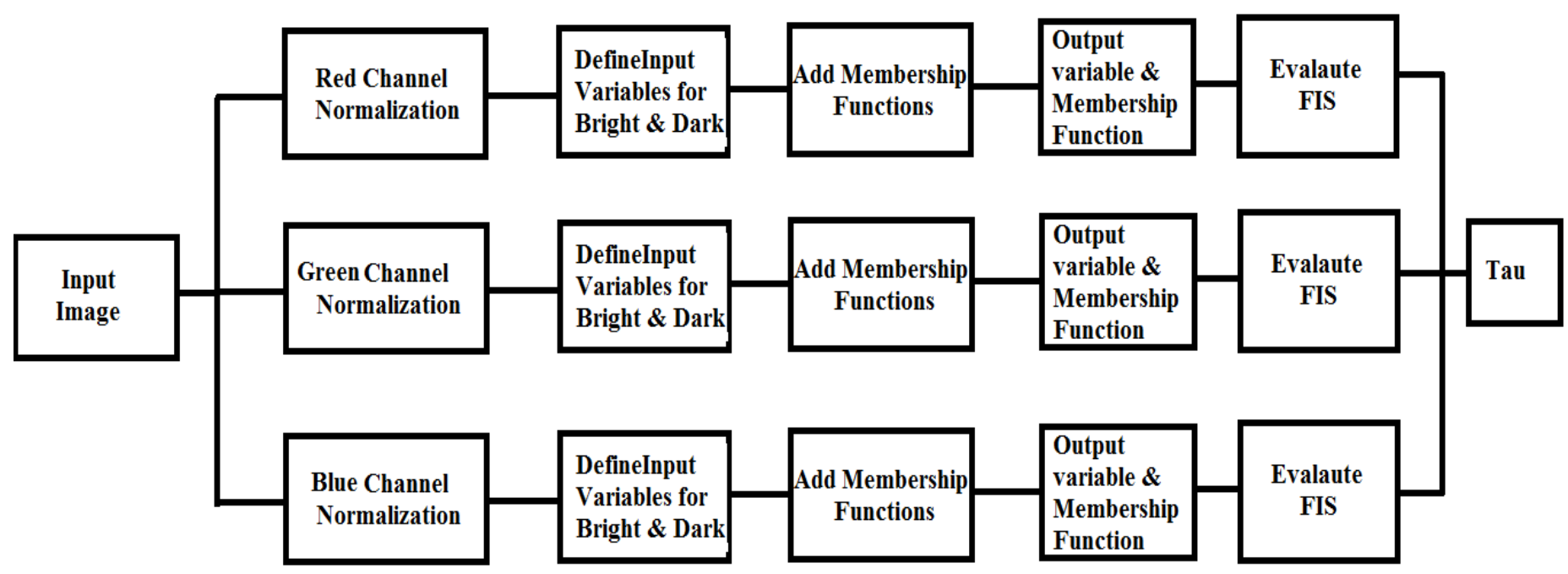

Figure 1. Automatic Fuzzy Intensification using Fuzzy Inference System

Algorithm: Automatic Fuzzy Intensification using Fuzzy Inference System

Step 1: Extract the Red, Green and Blue channel separately and normalize each channel for better input understanding by using equation (2)

$$
\mathrm{Ir}=\mathrm{Ir} / 255 ; \mathrm{Ig}=\mathrm{Ig} / 255 ; \mathrm{Ib}=\mathrm{Ib} / 255
$$

Step 2: Define variable for bright and dark intensities for each normalize channel by using equation (3) and (4)

$\mathrm{Td}=\operatorname{addvar}(\mathrm{Td},[\mathrm{Ir}, \mathrm{Ig}, \mathrm{Ib}] \mathrm{D},[\mathrm{low}, \mathrm{mid}])$ for dark

$\mathrm{Tb}=\operatorname{addvar}(\mathrm{Tb},[\mathrm{Ir}, \mathrm{Ig}, \mathrm{Ib}], \mathrm{B},[\mathrm{mid}+1$, high $])$ for bright (4)

Where low $=0$, mid $=0.5$ and high $=1$ 
Step 3: Add membership function for input defined variable by using Gaussian membership function by using equation (5) and (6)

$\mathrm{Td}=\operatorname{addmf}(\mathrm{Td},[\mathrm{Ir}, \mathrm{Ig}, \mathrm{Ib}], 1$, zero, gaussmf, [sx, mid]) (5)

$\mathrm{Tb}=\operatorname{addmf}(\mathrm{Tb},[\mathrm{Ir}, \mathrm{Ig}, \mathrm{Ib}], 2$,zero, gaussmf', [sy, high]) (6)

Step 4: define output variable and find out membership function by using equation (7) and (8)

Td = addmf $($ Td,output, 1, dark,trimf,[wa, wb, wc] $)$

$\mathrm{Tb}=\operatorname{addmf}(\mathrm{Tb}$, output, 1, bright,trimf, $[\mathrm{ba}, \mathrm{bb}, \mathrm{bc}])$

Where $\mathrm{wa}=0.0, \mathrm{wb}=0.2, \mathrm{wc}=0.4, \mathrm{ba}=0.6, \mathrm{bb}=0.7, \mathrm{bc}=$ 0.9 ;

Step 5: By using above parameter and Fuzzy inference system an appropriate intensification operator $\{\tau \mathrm{R}, \tau \mathrm{G}, \tau \mathrm{B}\}$ for the given image is calculated for each channel. Now by using $\{\tau R$, $\tau \mathrm{G}, \tau \mathrm{B}\}$, processed channel is calculated by formula (9)

$$
\mathrm{kIc}=\underset{2 *(\operatorname{fIc}(\llbracket \mathrm{x}, \mathrm{y})) \rrbracket \wedge(2) \text { if } \operatorname{fIc}(\mathrm{x}, \mathrm{y})<\tau \mathrm{c}}{1-2 *(\operatorname{fIc}(\llbracket \mathrm{x}, \mathrm{y})) \rrbracket \wedge(2) \text { otherwise }}
$$

Where, $\tau \mathrm{c}$ is $\{\tau \mathrm{R}, \tau \mathrm{G}, \tau \mathrm{B}\}$ for different colour channel and " $\mathrm{kIc}$ " is the processed channels by intensification operators for any channel red, green and blue. Formula (10) is used to observe the pixels of the output image.

$$
\mathrm{uc}=\llbracket(\mathrm{kIc}) \rrbracket \wedge(\tau \mathrm{c}+\xi)
$$

Where c represents any colour channel and " $\xi$ " is the intensification tuning parameter, varies between 0 and 1 . It

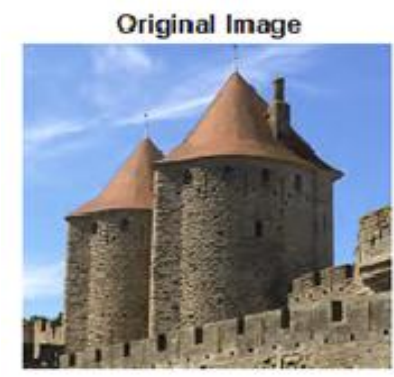

(a)

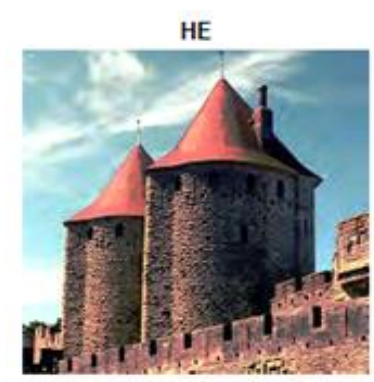

(g)

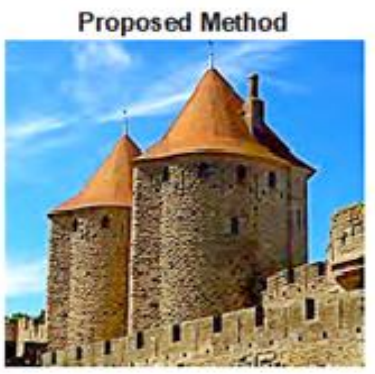

(b)

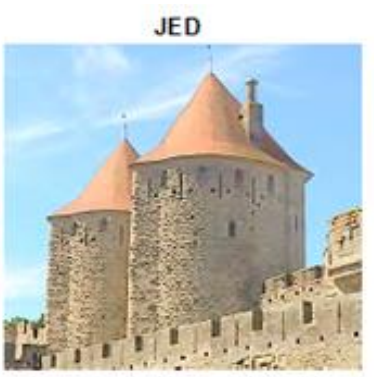

(h)

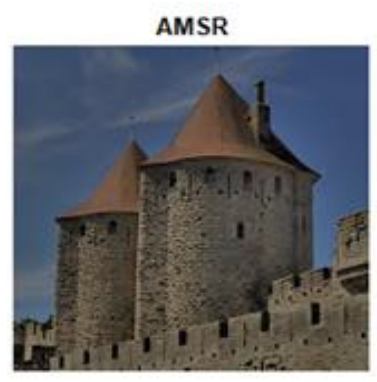

(c)

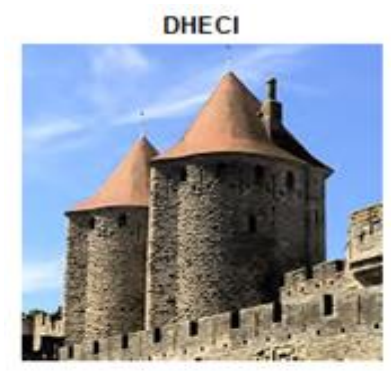

(e)

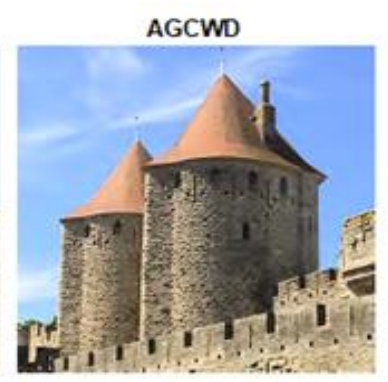

(d)

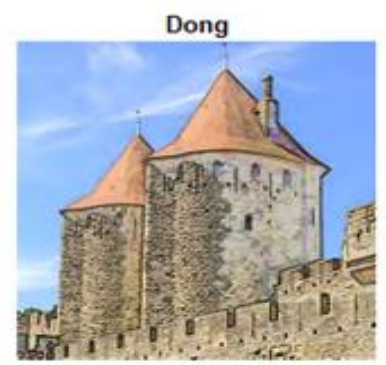

(f)

Figure 2. Results for img13 (a) Input Image (b) Proposed Method (c) AMSR (d) AGCWD (e) DHECI (f) Dong (g) HE (h) JED 
Table 1. Comparison of Different Techniques with Proposed Method

\begin{tabular}{|l|r|r|l|l|l|l|c|c|}
\hline & MSE & LOE & PSNR & SSIM & NIQE & Contrast & Colourfulness & Entropy \\
\cline { 2 - 8 } Original & & & & & & 10.5260 & 48.7390 & 7.3265 \\
\hline Proposed & 37.4409 & 82.2820 & 18.6187 & 0.7749 & 3.6940 & 10.8866 & 81.7955 & 7.3078 \\
\hline AMSR & 4.7638 & 435.7716 & 11.6483 & 0.7772 & 4.0572 & 9.3890 & 23.8431 & 6.4876 \\
\hline AGCWD & 64.3438 & 46.9328 & 20.1991 & 0.9572 & 3.8759 & 10.8924 & 52.5191 & 7.1480 \\
\hline DHECI & 36.0801 & 32.0804 & 22.9528 & 0.9421 & 3.9766 & 11.1567 & 53.1613 & 7.3212 \\
\hline DONG & 59.3594 & 352.6560 & 14.8907 & 0.8469 & 3.7049 & 10.3166 & 50.9585 & 7.0716 \\
\hline HE & 23.1822 & 192.8052 & 19.0545 & 0.6947 & 3.3519 & 10.8571 & 48.6205 & 5.9651 \\
\hline JED & 80.9166 & 253.8016 & 14.0340 & 0.8434 & 4.8640 & 10.6408 & 43.3156 & 6.5920 \\
\hline
\end{tabular}

Table 1 demonstrate the test results of comparing revised methodologies using MSE, LOE, PSNR, SSIM, NIQE, contrast, colourfulness and entropy.

The experiments were also performed on MATLAB R2018a on 10 actual images from a database obtained from the Urban Scenery data form for colour images. Following Figure 3 shows the images of the results obtained using the various available methods.

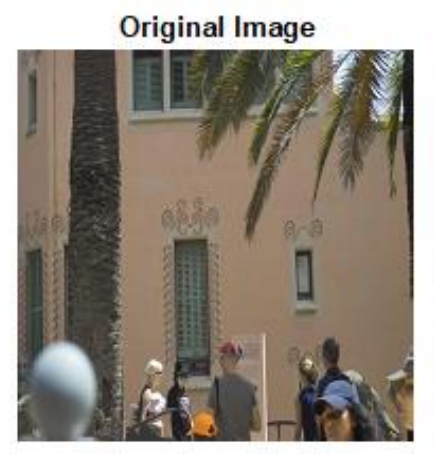

(a)

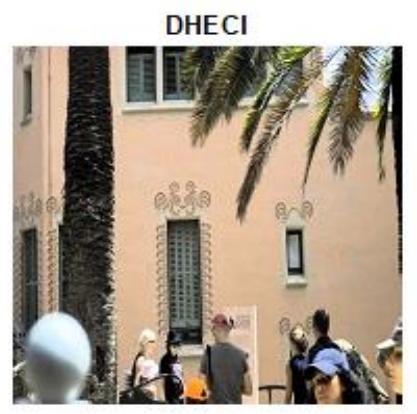

(e)

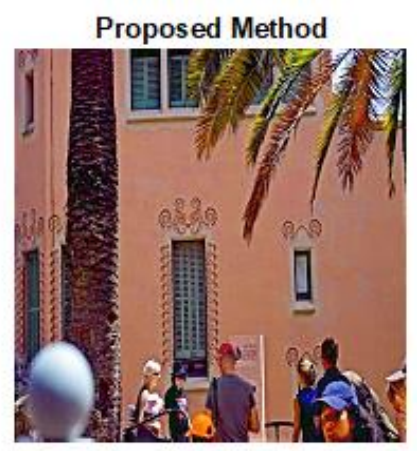

(b)

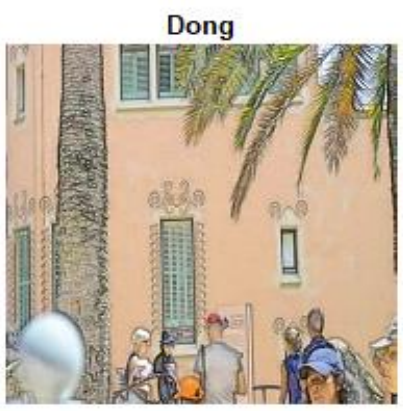

(f)

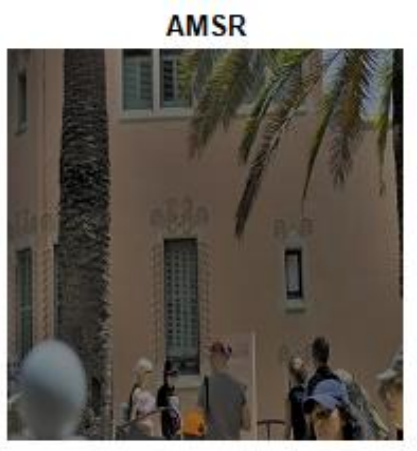

(c)

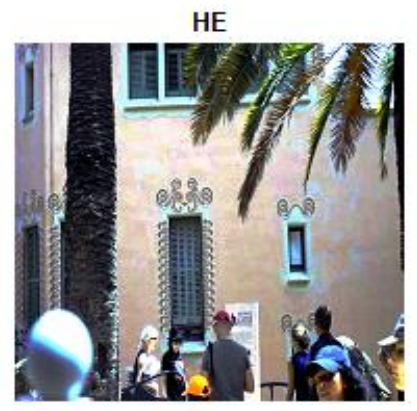

(g)

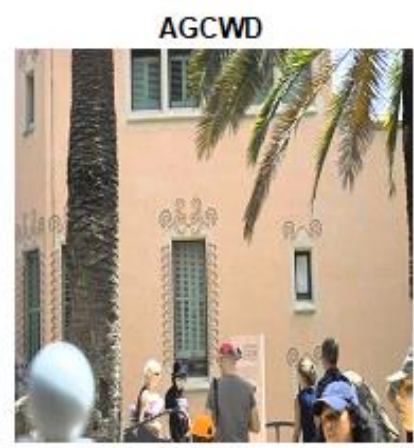

(d)

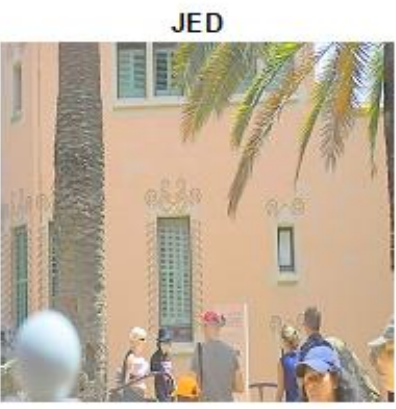

(h)

Figure 3. Results for usd 4 of USD Dataset (a) Original Image (b) Proposed Method (c) AMSR (d) AGCWD (e) DHECI (f) Dong (g) HE (h) JED 
Table 2. Comparison of Different Techniques with Proposed Method

\begin{tabular}{|l|r|r|l|r|r|r|c|c|}
\hline & \multicolumn{1}{|l|}{ MSE } & \multicolumn{1}{l}{ LOE } & PSNR & SSIM & NIQE & Contrast & Colourfulness & Entropy \\
\hline Original & & & & & & 10.3569 & 23.7515 & 6.8965 \\
\hline Proposed & 29.5374 & 131.4596 & 20.1210 & 0.7051 & 2.8649 & 10.4953 & 49.5988 & 7.0894 \\
\hline AMSR & 0.0102 & 355.5416 & 16.3706 & 0.8546 & 2.4873 & 9.5150 & 15.8797 & 6.3395 \\
\hline AGCWD & 77.6057 & 41.2244 & 14.3827 & 0.8705 & 2.8745 & 11.1813 & 34.5519 & 7.2977 \\
\hline DHECI & 58.8196 & 50.5416 & 15.3990 & 0.8355 & 2.8333 & 11.6178 & 34.0840 & 7.4268 \\
\hline DONG & 81.3361 & 361.1464 & 12.4782 & 0.7574 & 3.1734 & 10.6750 & 34.7852 & 6.9515 \\
\hline HE & 44.1605 & 168.2584 & 14.2906 & 0.6001 & 3.3643 & 10.7912 & 35.1711 & 5.8694 \\
\hline JED & 84.3921 & 121.7740 & 12.5098 & 0.8069 & 4.1674 & 10.3741 & 34.8888 & 6.7996 \\
\hline
\end{tabular}

Table 2, Fig. 5(a), 5(b), 5(c), 5(d), 5(e), 5(f), 5(g), and $5(h)$ show the test results of comparing various methodologies using MSE, LOE, PSNR, SSIM, NIQE, Contrast, Colourfulness, and Entropy parameters, as well as the corresponding graphs.

In this study, we used 15 test images from the CEED database in bmp format and 10 images from the Urban Scenery Database in jpeg format. All images are very different in strength, ready to test the feasibility of the proposed method. Subjective testing is performed on 15 CEED and 10 USD images. The proposed method is the most effective in this investigation since it improves brightness and colour contrast in all types of photos while maintaining brightness.

In the targeted quality test the analysis contained 15 CEED and 10 USD images of eight quality parameters (MSE, LOE, PSNR, SSIM, NIQE, Contrast, Colourful and Entropy). Now here we are with the image effects in Figure 2 (img13). This measurement was used to compare all processing methods: Histogram Equalization [24], AGCWD [9], DHECI [25], Dong [27], AMSR [28], and JED [29]. In terms of several quality criteria, the suggested method is also compared to existing techniques in objective assessment, as indicated in Table 1 of img13. Mean Square Error values of the proposed route are below all values except for one AMSR which shows the maximum interaction with the original image. Lightness Order Error LOE is much lower than AMSR, Dong, HE and JED which shows least lightness error in proposed algorithm. The PSNR values of imgl3 are also better compared to many alternatives other than AGCWD and DHECI. The suggested method's SSIM values are better than HE only whereas the NIQE values are better than all other approaches, showing just some commitment to natural image quality. The image contrast is better than AMSR, Dong, HE and JED which shows that better comparison of the proposed method. The brightness in Table 1 is higher than all the methods used which indicates that the proposed method incorporates information.
The entropy value of the proposed route image is better than JED, HE, Dong, AGCWD, AMSR but lower than DHECI which indicated the proposed route produces a better image.

Now here we are presenting the results of images in Figure 3 (usd4). In objective evaluation the proposed method is also compared with present methods when it comes to several excellence metrics as shown in Table 2 of the usd4 image. The MSE values for the projected method is lower than all methods except one AMSR representing greater association with the input image. Lightness Order Error LOE is much lower than AMSR, Dong, and HE which shows lesser lightness error in proposed algorithm. PSNR output of the img13 is also enhanced as matched to available techniques which shows better signal component in perceived image. SSIM found for proposed technique is improved than HE only where as NIQE found are superior to AGCWD, Dong, HE and JED whereas slightly greater than DHECI and AMSR which shows that there is least deviation in natural image quality. Contrast of image is higher than AMSR, HE and JED and slightly lower than other methods showing that better contrast of proposed method. Colourfulness in Table 2 is higher than all methods used which shows that proposed method enriches the colour information. Entropy value of projected method is also improved than JED, HE, Dong, AMSR but lower than DHECI and AGCWD which shown the proposed method produces image having good contents.

Analysis of Table 1 and Table 2 shows a visual representation as a quality measure. Other methods better brightness values comparing with metrics namely PSNR, SSIM, NIQE, MSE, LOE and Entropy do not have a positive perception.

Visible compared to the proposed method. There is a trade-off among achievement measures and ability to view quality again, as shown in this study. As a result, the proposed method significantly outperforms the competition in terms of both design and measurement metrics. 
Table 3. Execution Time of different images

\begin{tabular}{|c|c|c|c|}
\hline \multirow[b]{3}{*}{ Methods } & \multicolumn{3}{|c|}{ Image } \\
\hline & $\operatorname{Img} 13$ & Img2 & USD4 \\
\hline & & & \\
\hline Proposed & 5.050 & 0.997 & 0.845 \\
\hline AMSR & 0.834 & 0.412 & 0.391 \\
\hline AGCWD & 0.188 & 0.100 & 0.072 \\
\hline DHECI & 15.628 & 15.433 & 15.218 \\
\hline DONG & 0.485 & 0.399 & 0.373 \\
\hline HE & 0.063 & 0.024 & 0.010 \\
\hline ZED & 7.499 & 7.101 & 7.245 \\
\hline
\end{tabular}

Figure 6 Shows the graphical chart for execution time of different images using different methods along with proposed method.

Table 3 shows the cost of computation in the proposed and available techniques for three randomly selected images. The duration taken of the proposed method for Imgl3 image is lower than the DHECI and JED methods which are slightly higher than other methods. In Img-2, the performance time is often lower than DHECI and JED where as it is slightly larger than the other method and identical for the usd4 image. Although HE takes very little time to do so many of his barriers force him to ignore this approach. By evaluating the performance of quality and quantity the proposed algorithm offers satisfactory results in various studies and also works well in terms of execution time.

\section{CONCLUSIONS}

An excellent colour contrast algorithm programme with local LCC and enhanced fuzzy intensification is used in this work to improve viewing quality and retain crucial image material. The proposed approach addresses both the confined minima and comprehensive maxima complications. To stunned the difficulties and introduce the right enhancement method, The RGB colour space is converted to HSL colour space first. LCC is employed in the HSL colour space's luminance component to improve image contrast depending on the adjacent pixels. The overexposed and no exposed parts have been well brightened, and the obtained images exhibit sharp contrast. Intensification operators are retrieved using a fuzzy inference technique, which is employed to regulate the hue conformity of the native hue changed pixels. The method has been applied and verified on CEED and USD databank images, as well as many exiting strategies. Although projected method takes a little more execution time in comparison to some methods but delivers agreeable output and formed natural contrast images with least or no artifacts and outclassed the many present $\mathrm{CE}$ techniques while comparing with quality parameters like MSE, LOE, PSNR, SSIM, NIQE, Contrast, Colourfulness and Entropy image evaluation parameters.

Author Contributions: The main research and analysis was provided by Avadhesh Kumar Dixit. The supervision and revision was provided by Rakesh Kumar Yadav and Ramapati Mishra.".

Acknowledgments: I express my sincere gratitude towards Dr. Rakesh Kumar Yadav, Assistant Prof. Department of Computer Science and Engineering IFTM University Moradabad and Professor Ramapati Mishra, Department of Electronics and Communication Engineering, Dr. RML Avadh University Ayodhya for his valuable suggestions and guidance. I also pay thanks to Head of Department of Computer Science and Engineering IFTM University, Moradabad for providing us necessary facilities for the implementation of this research work. 
Conflicts of Interest: "The authors declare no conflict of interest"

\section{REFERENCES}

[1] Gonzales R.C. and R.E. Woods, Digital Imaging Process. Prentice Hall, New Jersey, 2002.

[2] S. Sridhar, Digital Image Processing. Oxford University Press, New Delhi, 2013.

[3] A. A. Michelson, Studies in Optics. Chicago: USA: University of Chicago Press, 1927.

[4] E. Peli, "Contrast in complex images," J. Opt. Soc. Am. A, vol. 7, no. 10, pp. 2032-2040, 1990.

[5] G. Yadav, S. Maheshwari, and A. Agarwal, "Contrast limited adaptive histogram equalization based enhancement for real time video system," in 2014 International Conference on Advances in Computing, Communications and Informatics (ICACCI), 2014, pp. 2392-2397.

[6] M. Abdullah-Al-Wadud, M. Kabir, M. Dewan, and O. Chae, "A Dynamic Histogram Equalization for Image Contrast Enhancement," Consum. Electron. IEEE Trans., vol. 53, pp. 593-600, Jun. 2007.

[7] S. Chien, F. Chang, K. Hua, I. Chen, and Y. Chen, "Contrast enhancement by using global and local histogram information jointly," in 2017 International Conference on Advanced Robotics and Intelligent Systems (ARIS), 2017, p. 75.

[8] M. Moniruzzaman, M. Shafuzzaman, and M. Hossain, Brightness preserving Bi-histogram equalization using edge pixels information. 2014.

[9] H. Ibrahim and N. S. P. Kong, "Brightness Preserving Dynamic Histogram Equalization for Image Contrast Enhancement,” IEEE Trans. Consum. Electron. vol. 53, no. 4, pp. 1752-1758, 2007.

[10] L. Zhuang and Y. Guan, "Image Enhancement via Subimage Histogram Equalization Based on Mean and Variance," Comput. Intell. Neurosci., vol. 2017, p. 6029892, 2017.

[11] S. Der Chen and A. Ramli, "Minimum mean brightness error Bihistogram equalization in contrast enhancement," Consum. Electron. IEEE Trans., vol. 49, pp. 1310-1319, Dec. 2003.

[12] G. Raju and M. S. Nair, "A fast and efficient colour image enhancement method based on fuzzy-logic and histogram," AEU - Int. J. Electron. Commun., vol. 68, no. 3, pp. 237-243, 2014.

[13] S. C. F. Lin et al., "Image Enhancement Using the Averaging Histogram Equalization (AVHEQ) Approach for Contrast Improvement and Brightness Preservation," Comput. Electr. Eng., vol. 46, no. C, pp. 356-370, Aug. 2015.

[14] C. Wong et al., "Histogram Equalization and Optimal Profile Compression based Approach for Colour Image Enhancement," J. Vis. Commun. Image Represent. vol. 38, Apr. 2016.

[15] G. Cao, L. Huang, H. Tian, X. Huang, Y. Wang, and R. Zhi, "Contrast enhancement of brightness-distorted images by improved adaptive gamma correction," Comput. Electr. Eng., vol. 66, pp. 569-582, 2018.

[16] S. Zaheeruddin and K. Suganthi, "Image Contrast Enhancement by Homomorphic Filtering based Parametric Fuzzy Transform," Procedia Comput. Sci., vol. 165, pp. 166-172, 2019.

[17] B. Subramani and M. Veluchamy, "Quadrant dynamic clipped histogram equalization with gamma correction for colour image enhancement," Colour Res. Appl., vol. 45, no. 4, pp. 644-655, 2020.

[18] R. Chandrasekharan and M. Sasikumar, "Fuzzy Transform for Contrast Enhancement of Nonuniform Illumination Images," IEEE Signal Process. Lett., vol. 25, no. 6, pp. 813-817, 2018.

[19] A. K. Dixit, R. K. Yadav, and R. Mishra, "Image Contrast Optimization using Local Colour Correction and Fuzzy Intensification," Int. J. Adv. Comput. Sci. Appl., vol. 12, no. 5, pp. 115-124, 2021.

[20] A. (2017) Qureshi, Muhammad Ali; Sdiri, Bilel; Deriche, Mohamed; Alaya-Cheikh, Faouzi; Beghdadi, “'Contrast Enhancement Evaluation Database (CEED2016)', Mendeley Data," vol. 3, 2017.

[21] J. Yang, W. Zhong, and Z. Miao, "On the Image enhancement histogram processing," in 2016 3rd International Conference on Informative and Cybernetics for Computational Social Systems (ICCSS), 2016, pp. 252-255.

[22] K. Nakai, Y. Hoshi, and A. Taguchi, "Colour image contrast enhacement method based on differential intensity/saturation graylevels histograms," in 2013 International Symposium on Intelligent Signal Processing and Communication Systems, 2013, pp. 445-449.

[23] X. Dong, Y. Pang, and J. Wen, "Fast efficient algorithm for enhancement of low lighting video," 2011 IEEE Int. Conf. Multimed.
Expo, pp. 1-6, 2011.

[24] C.-H. Lee, J.-L. Shih, C.-C. Lien, and C.-C. Han, Adaptive Multiscale Retinex for Image Contrast Enhancement. 2013.

[25] X. Ren, M. Li, W. H. Cheng, and J. Liu, "Joint Enhancement and Denoising Method via Sequential Decomposition," Proc. - IEEE Int. Symp. Circuits Syst., vol. 2018-May, 2018.

[26] S. Wang, J. Zheng, H.-M. Hu, and B. Li, "Naturalness Preserved Enhancement Algorithm for Non-Uniform Illumination Images," IEEE Trans. Image Process. vol. 22, May 2013.

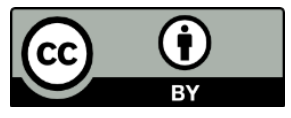

(C) 2021 by the Avadhesh Kumar Dixit, Rakesh Kumar Yadav and Ramapati Mishra Submitted for possible open access publication under the terms and conditions of the Creative Commons Attribution (CC BY) license (http://creativecommons.org/licenses/by/4.0/). 